\title{
An Algorithm to Realize the Real-time Interpolation of Bezier Curves Based on DSP
}

\author{
Yujie Zhang, Weijie Zhang, Bin Xu, Chang Liu \\ North China Institute of Science and Technology \\ NCIST YanJiao, CIty of LangFang, HeBei province, China
}

\begin{abstract}
As the surface of parts to be shaped is complex, there is a high expectation on accuracy and smoothness during CNC machining. One way is proposed to realize the surface of complex parts based on bezier curves, which is with the advantages of realizing the surface of complex parts in an accurate way. Firstly, the surface of complex parts should be represented by bezier curves; secondly, the bezier curves should be cut in the same length; at last, the pulse signal should be sent to each axis. The experimental results demonstrate that the surface of complex parts which has been shaped meets the expectation on accuracy and smoothness.
\end{abstract}

\section{Keywords-DSP; stepper motor; bezier curves; interpolation}

\section{I.Introduction}

In the processing of complex shape workpiece of $\mathrm{CNC}$ machine tools, such as submarine propeller,not linear interpolation generally meet the requirements of circular interpolation. Curve geometric properties of Bezier is fine, continuous two order derivatives, appear not shocks in the linear transition. ${ }^{[1]}$

This paper presents A method of uniform cutting Bezier curve, so that the smooth running of the motor, effectively solve the frequent acceleration and deceleration, and avoid stepping motor slow speed response, and the problem of low frequency oscillation. At the same time to control the cumulative errors of fitting process and chord error, so as to ensure the processing performance of the machine. ${ }^{[2]}$

\section{II.control algorithm of Bezier curve}

In the three-dimensional Euclidean space, given $\mathrm{n}+1$ points $P_{0} 、 P_{1} \cdots \cdots P_{n}$, known as data points, and the connection of these points to form the control polygon, with the start of the control polygon and the end of the two side tangent. shown in Figure1.

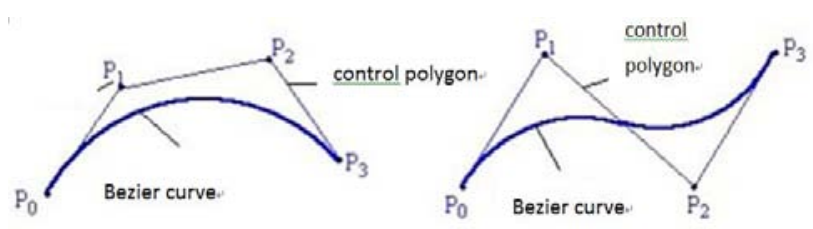

Figure 1 Bezier curve and control polygon

The trajectory equation of Bezier curve expressed as (1), $\mathrm{t}$ is a Bezier curve control variable, varying between 0 to 1 . While $\mathrm{t}=0$, the curve start, While $\mathrm{t}=0$, the curve end, more classification division of $\mathrm{t}$,more points on the Bezier curve , the change of $\mathrm{T}$ in the interval of $[0,1]$, to form Bezier curve. ${ }^{[3]}$

$$
P_{(t)}=\sum_{i=0}^{n} P_{i} J_{i, n(t)} \quad i=0,1 \ldots \ldots n
$$

$p_{i}$ is the No.i coordinates of the point, ${ }^{J, t}$ is known as the Bernstein basis function, expression is as follows

$$
\begin{gathered}
J_{i, t}=C_{n}^{i} t^{i}(1-t)^{n-i} \\
C_{n}^{i}=\frac{n !}{i !(n-i) !} \quad i=0,1 \ldots \ldots n \\
p_{x(t)}=\sum_{i=0}^{3} p_{x(i)} J_{i, t}=\sum_{i=0}^{3} p_{x(i)} C_{3}^{i} t^{i}(1-t)^{3-i} \\
=\frac{n !}{i !(n-i) !} \sum_{i=0}^{3} p_{x(i)} t^{i}(1-t)^{3-i}
\end{gathered}
$$




$$
=\left[\begin{array}{llll}
t^{3} & t^{2} & t & 1
\end{array}\right]\left[\begin{array}{cccc}
-1 & 3 & -3 & 1 \\
3 & -6 & 3 & 0 \\
-3 & 3 & 0 & 0 \\
1 & 0 & 0 & 0
\end{array}\right]\left[\begin{array}{c}
p_{x 0} \\
p_{x 1} \\
p_{x 2} \\
p_{x 3}
\end{array}\right]
$$

$\mathrm{t}$ changes between 0 to 1 , Bezier curve can be drawn in the space, the operation of the motor of a complete Bezier curve takes time $T$, so there is a proportional relationship between $t \mathrm{~T}$ and $\mathrm{T}, T=a t$ (a is a constant). A small section of the whole section curve of the motor operating assumptions, $t$ is variable from $t_{(i-1)}$ to $t_{i}$, by the time point from $T_{(i-1)}$ to $T_{i}$, last for $\Delta T_{i}$, and $\Delta T_{i}=T_{i}-T_{(i-1)}$.

In order to solve the problem of burr parts and machine tremor, to achieve a smooth Bezier curve fitting, stepper motor control every step of the step. $t_{0}=0$, set the start point. The interpolation point value after respectively $t_{1} 、 t_{2}, t_{3} \cdots \cdots t_{n}$ $\left(t_{n}=1\right)$, set the corresponding point $Q_{1} 、 Q_{2} 、 Q_{3} \ldots \ldots Q_{n}$, coordinate points on the curve in $\mathrm{X}, \mathrm{Y}, \mathrm{Z}$ direction is $q_{x k}$ 、 $q_{y k}, q_{z k}$. Since the set of step motor in equal distance movement in each unit time interval, while the time and the curve of control variable is a linear relation, expressed as $T=a t\left(a\right.$ is a constant), so the variable $t$ in $\Delta t_{1}=t_{1} t_{1}-t_{0}$, $\Delta t_{2}=t_{2}-t_{1}, \Delta t_{3}=t_{3}-t_{2} \cdots \cdot \Delta t_{n}=t_{n}-t_{n-1}$, the distance $L_{k}$ is the same, expressed as (5) (6):

$$
\begin{aligned}
& L_{1}=\sqrt{\left(q_{x 1}-q_{x 0}\right)^{2}+\left(q_{y 1}-q_{y 0}\right)^{2}+\left(q_{z 1}-q_{z 0}\right)^{2}} \\
& \quad \cdots \ldots \\
& L_{n}=\sqrt{\left(q_{x n}-q_{x(n-1)}\right)^{2}+\left(q_{y n}-q_{y(n-1)}\right)^{2}+\left(q_{z n}-q_{z(n-1)}\right)^{2}}
\end{aligned}
$$

$L_{1}, L_{2} \cdots \cdots L_{\mathrm{n}}$ express distance value of the section1 to the section $\mathrm{n}$.

In order to realize each section of step motor, then $L_{1}=L_{2}=L_{3}=L_{4}=\cdots \cdots L_{\mathrm{n}}$, Each segment, calculated value of $t_{k}$, first determine the value of $\mathrm{t} 1 \quad\left(t_{0}=0, t_{n}=1\right)$, the calculated value $L_{1}$, the other value $t_{n}$ can be calculated sequentially. Coordinate Bezier path described above before every point of $\mathrm{X}, \mathrm{Y}, \mathrm{Z}$ direction is controlled by the value of $t_{k}$ variables are identified, then you can get the absolute coordinates of each point value, also can calculate the relative coordinates, the amount of exercise to control each axis stepper motor with each step, the concrete solving the third section steps reference.

\section{III.Error analysis of real-time interpolation}

In order to guarantee the Bezier curve is not distortion, using the method of position control, line segment Bezier curve can be short connected to approach, and in which every interpolation points on the theory of Bezier curve,so there is no accumulated error. Stepper motor in each step of operation is equidistant. But because the Bezier curve is discretization, the distance between Bezier curve in the middle point and the actual: chord error, as shown in figure $2:^{[4]}$

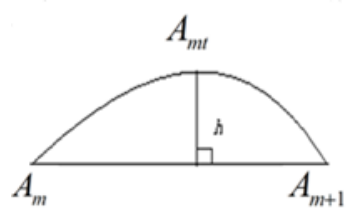

figure 2 Chord error

Arbitrarily two interpolation points $A_{m}$ and $A_{m+1}, \mathrm{~m}$ refers to the No.m interpolation points, above the arc is Bezier curve theory, $A_{m t}$ is a point on the curve, straight line between $A_{m}$ and $A_{m+1}$ the actual trajectory is approximate fitting 
stepper motor. There is a distance between the curves of $A_{m} A_{m t} A_{m+1}$ and linear $A_{m} A_{m+1}$, namely the chord error.

The highest point of the $A_{m} A_{m t} A_{m+1}$ curve to the straight line distance of $A_{m} A_{m+1}$ expressed as $h_{m}$, chord error calculation formula expressed as (11):

Set motion to $A_{m}$ and $A_{m+1}$, twere $t_{m}$ and $t_{m+1}, t_{j}$ said the definition of an arbitrary value between $t_{m}$ and $t_{m+1}$, that is $\mathrm{t}$ in $\left[t_{m}, t_{m+1}\right]$. Set the $a_{x(m+1)}$ and $a_{x m}$ are respectively $A_{m}$ and $A_{m+1}$ in the $\mathrm{X}$ direction of the coordinate direction, said X Bezier theory on the curve between $A_{m}$ and $A_{m+1}$ a little $A_{m t}$ coordinates, $h_{x m}$ said $A_{m t}$ to the actual trajectory $\mathrm{X}$ direction distance, such as type (8). In $\mathrm{Y}$ and $\mathrm{Z}$ direction are indicated and so on, such as the type (9) (10). A practical Bezier curve of the above point $A_{m t}$ to approximate a straight line segment distance is $h_{m t}$, the calculating formula of $h_{m t}$ such as (8):

$$
\begin{aligned}
& h_{x m t}=\sqrt{\left(\left(\left(\frac{t_{j}-t_{m}}{t_{m+1}-t_{m}}\left(a_{x(m+1)}-a_{x m}\right)+a_{x m}\right)-a_{x m t}\right)^{2}\right.} \\
& h_{m t}=\sqrt{{h_{x m}^{2}+h_{y m}^{2}+h_{z m}^{2}}^{2}}
\end{aligned}
$$

Bow height error $h_{m}$ is the maximum value of $h_{m t}$, if the distance between $A_{m} A_{m+1}$ is very small (approaching 0), bow high error $h_{m}$ can be controlled in a certain range. Value of real-time calculation at the time of the chord error in the interpolation process, can improve the error, but the chord error may appear arbitrary between the a bit, if every step of the calculation, it requires a great deal of calculation, is very memory, affect the real-time interpolation. So, through an example, the value of the coordinates of the point are A set of four type A $(1,2,2), B(4,8,7), C(6,15,9), D(9,18,3)$, the $t$ is divided into 1000 parts, namely $n=1000$, a curve segment selected segmented, through the MATLAB results calculated, straight-line distance of two interpolation points to is represented as a calculated, $k_{a}=0.02301$, setting $m=526$ (526th interpolation points), and then computing in many times in the distance $k_{a}\left(t_{j}\right.$ changes in $\left.\left[t_{m}, t_{m+1}\right]\right)$, then $h_{m}=0.003, \quad h_{m}=0.13 k_{a}$, the chord error value $h_{m}$ is very small, far less than $k_{a}$, can be ignored.

IV.Real-time interpolation steps of cubed Bezier curve

The real-time interpolation steps described as the 8 steps:

1. According to given points, calculate the Bezier curve.

2. $t_{0}=0$, set the value of $t_{1}$, the value of $L_{1}$ is the length of a step to be calculated.

3. $n=n+1$ ( $n=1$ at the beginning), calculate $t_{n}$ of every step according to $L_{n}=L_{1}$.

4.if $t_{n}<1$, according to $t_{n}$ of step $\mathrm{n}$, calculate the every step of real-time $\mathrm{x}, \mathrm{y}, \mathrm{z}$ coordinates, as $p_{x n}, p_{y n}, p_{z n}$.

5.calculate the coordinate difference between last step of $\mathrm{x}$, $\mathrm{y}, \mathrm{z}$, as $: p_{x n}-p_{x(n-1)}, \quad p_{y n}-p_{y(n-1)}, \quad p_{z n}-p_{z(n-1)}$.

6.get the feed rate of each stepper motor in the $x, y, z$ direction, converse it into pulse sent to stepper motor. 
7 when $t_{n}>1$, set $t_{n}=1$, go on step5, 6,8 .

8 , the end.

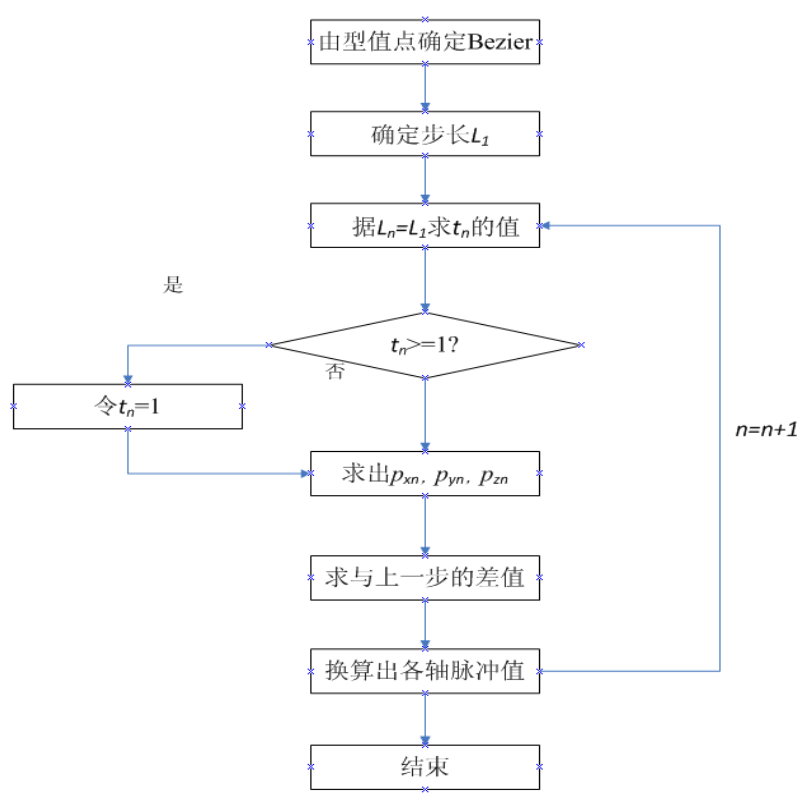

Figure3 steps of interpolation

V.interpolation and a case

Each step is a spatial curve, straight line in space length longer than or any $\mathrm{x}, \mathrm{y}, \mathrm{z}$ and in a direction along the road. Set the step motor to a certain speed to go the step distance, time is $T_{C}, T_{C}=C / v$. because of the space curve in $\mathrm{x}, \mathrm{y}, \mathrm{z}$ direction, $C_{\mathrm{x}} 、 C_{\mathrm{y}} 、 C_{\mathrm{z}}$ is not the same, and $C_{x} \leq C 、 C_{y} \leq C$ 、 $C_{z} \leq C$, so the pulse number of each motor is different. Calculate each step of each shaft speed, $v_{x}=C_{x} / T_{C}$ 、 $v_{y}=C_{\mathrm{y}} / T_{C} 、 v_{z}=C_{z} / T_{C}$, real-time transmission to each axis, in order to ensure uniform rate movement.
According to the steps described in the previous section, 1000 interpolation points are set, a case shown in Fig. 4.

Four coordinate interpolation points are respectively A (6, $1,2), \mathrm{B}(4,8,7), \mathrm{C}(3,8,1), \mathrm{D}(9,18,3)$, interpolation cycle is $0.01 \mathrm{~s}$, the process of continuous $10 \mathrm{~s}$, each step of $0.2 \mathrm{~mm}$, interpolation speed $20 \mathrm{~mm} / \mathrm{s}$. Through the simulation curves we can see that the curve is smooth, also control the chord error.

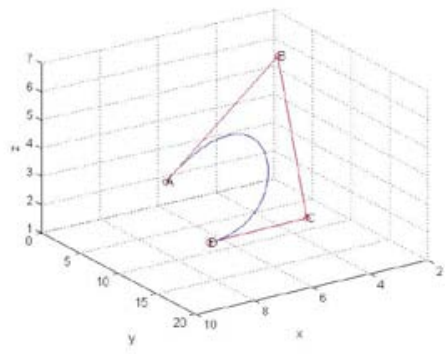

Figure 4 curves from different angel

IV. Conclusion

Under the control of DSP, each step of the step is certain, so the motor can smoothly make a uniform rate curve motion. By the high speed operation performance of DSP to ensure the real time control, real time through DSP sent to the $\mathrm{x}, \mathrm{y}$, zaxis of different pulses. To realize multi axis, this paper proposes a control method, to solve the $\mathrm{z}, \mathrm{y}, \mathrm{z}$ axis and uniform motion problems, ensure the curve distortion. The motor is running smoothly, to meet the processing requirements. ${ }^{[4]}$

\section{REFERENCES}

[1] Wang Youmin. Bezier curve trajectory optimization and control of continuous trajectory control of robot. The mechanical transmission of [J], 2003, 27 (3): 42-47.

[2] Kang Xincai. Knife Bezier surface NC machining triangular path generation and NC milling simulation, 2011 master's degree thesis of Shandong University of Technology.

[3] Yang Linying; splicing and extended [D] B zier curves; Northwest Normal University; 2013

[4] Golden E Herin. Next generation controller [J]. Morden Machine Shop, 2008, 63 (7):148- 152 